REVISTA PERUANA DE INVESTIGACIÓN EDUCATIVA 2021, No.15, pp.9-26

\title{
Las escuelas segregadas son el mensaje
}

\section{Manuel Bello}

Universidad Peruana Cayetano Heredia

manuel.bello@upch.pe

Recibido: $14 / 10 / 2021$

Aprobado: 27/11/2021 


\section{Las escuelas segregadas son el mensaje}

\section{Resumen}

La segregación escolar por niveles socioeconómicos (NSE) opera agrupando a los estudiantes de familias similares en pobreza o en riqueza. En el Perú, la segregación escolar por NSE ha crecido mucho en las últimas décadas, impulsada por dinámicas de mercado en la educación. Diversos estudios han encontrado que el nivel de pobreza de los estudiantes de cada escuela se asocia de manera relevante con la calidad de las condiciones escolares y con los resultados en evaluaciones de logros de aprendizaje. En este artículo, se revisa y discute cómo el carácter segregado y la ubicación de cada escuela en la pirámide estratificada por NSE que compone el sistema escolar peruano opera como un mensaje implícito y tiene efectos en las familias, en los estudiantes y en la sociedad. La composición social es uno de los criterios que aplican las familias para seleccionar la escuela para sus hijos, junto con algunas características relacionadas con las expectativas del grupo social al que pertenecen o aspiran a pertenecer. Para los estudiantes, el carácter segregado de su escuela es parte del currículo oculto, que propicia determinadas representaciones, actitudes y valores, diferentes para cada NSE. Este tiende a ocultar o naturalizar las desigualdades, justificar los privilegios, reducir las expectativas de los jóvenes más pobres, además de reducir la cohesión social, aumentar la intolerancia y la discriminación de aquellos que son diferentes. A partir de este estudio, se recomienda profundizar la investigación sobre los efectos de la vivencia de la segregación por NSE, que ahora prevalece sobre las intenciones democratizadoras del currículo escolar oficial.

Palabras clave: discriminación educacional, democratización de la educación, agrupamiento educacional, escuela, educación ciudadana 


\title{
Segregated Schools Are the Message
}

\begin{abstract}
School segregation by socioeconomic status (SES) operates by grouping students from families similar in poverty or wealth. In Peru, school segregation by SES has grown significantly in recent decades, driven by market dynamics in education. Several studies have found that the level of poverty of students in each school is associated with the quality of school conditions and with the results in evaluations of learning achievements. This article reviews and discusses how the segregated condition and the location of each school in the SES stratified pyramid that is the Peruvian school system operates as an implicit message and has effects on families, students, and society. Social composition is one of the criteria that families use to select the school for their children, along with some characteristics related to the expectations of the social group to which they belong or aspire to belong. For students, the segregated condition of their school is part of the hidden curriculum, which fosters certain representations, attitudes, and values, different for each SES; it tends to hide or naturalize inequalities, justify privileges, reduce the expectations of the poorest young people, in addition to reducing social cohesion, increasing intolerance and discrimination of those who are different. It is recommended to deepen research on the effects of the experience of segregation by SES, which now prevails over the democratizing intentions of the official school curriculum.
\end{abstract}

Keywords: educational discrimination, democratization of education, educational grouping, school, citizenship education 
El propósito de este artículo es analizar y discutir el rol de la segregación escolar como mensaje para las familias y los estudiantes, así como su efecto sobre el desarrollo de la identidad personal y social, la ciudadanía y el sentido de pertenencia a la sociedad. Parafraseando a McLuhan (1996), se puede decir que la escuela afecta a la sociedad no solo por los contenidos que transmite, sino también por sus propias características como institución; se asume que la escuela, en sí misma, es el mensaje. En este caso, se discute cómo la escuela, por su carácter selectivo y segregador por niveles socioeconómicos, es en sí misma un mensaje que opera como un contenido del currículo oculto de la educación (Giroux,1997), y que influye negativamente en los estudiantes y en todos los actores de la comunidad educativa, obstaculizando el desarrollo de la ciudadanía democrática prevista en el currículo oficial.

\section{Relevancia de la segregación escolar socioeconómica}

La segregación escolar es la distribución de los estudiantes en escuelas separadas, tomando como criterio de selección alguna característica personal, familiar o social. Cuando el criterio es el género, se separa a los estudiantes en colegios para varones y colegios para mujeres. Cuando se consideran supuestas dificultades o facilidades para aprender, se les separa en escuelas "especiales" o en "colegios de alto rendimiento". También, se separa a los estudiantes en función de la lengua materna, la opción religiosa o el nivel socioeconómico. Este último tipo de segregación escolar es el foco de atención del presente artículo.

La segregación escolar puede ser resultado de decisiones normativas, como las escuelas separadas por género o por discapacidades. Puede responder a preferencias de las familias, como cuando se separa por creencias religiosas. La segregación socioeconómica, en cambio, está asociada al poder económico o social de las familias, y opera agrupando a los estudiantes en función de la capacidad de pago o de retribución de cada familia por el servicio educativo que reciben sus hijos. En este caso, las escuelas agrupan mayoritariamente a estudiantes de condición socioeconómica similar (Murillo y Carrillo, 2021).

Varias investigaciones con datos del Llece-Unesco, de PISA, de ECEUMC, de Niños del Milenio y otros estudios han confirmado, usando diversos procedimientos de medición, que el sistema escolar peruano actual está integrado mayoritariamente por escuelas con una composición social homogénea, organizadas en una estructura estratificada y piramidal, que tiene en la punta a una pequeña cantidad de escuelas para ricos; y, en la base, a muchas escuelas para los hijos e hijas de las familias en situación de pobreza o pobreza extrema (Bello y Villarán, 2004; Benavides, León y Etesse, 2014; Murillo et al., 2018; Balarin y Escudero, 2019; Murillo y Carrillo, 2020 y 2021)

Las investigaciones internacionales y nacionales han mostrado que la segregación escolar por nivel socioeconómico (NSE) se asocia con la calidad de las condiciones para la enseñanza y para el aprendizaje, con las oportunidades para aprender y con los resultados del aprendizaje de los objetivos curriculares. 
Casi siempre, las escuelas que concentran una mayor cantidad de estudiantes pobres ofrecen una educación de menor calidad y obtienen los peores resultados en las evaluaciones de logros del aprendizaje (Mijs y Roe, 2021; Murillo y Carrillo, 2021). En otras palabras, la segregación escolar por NSE reproduce las desigualdades educativas (Carrillo, 2021).

La Oficina de Medición de la Calidad de la Educación (Ministerio de Educación [Minedu], 2019) identificó que, a medida que aumentaba la pensión en las escuelas privadas peruanas, mejoraban los resultados de aprendizaje: que los estudiantes de escuelas privadas con las pensiones más bajas tenían resultados similares o menores a los de sus pares de escuelas públicas, mientras que los estudiantes de colegios no estatales de alto costo obtenían los resultados más altos. En esa evaluación, se encontró que el mayor diferenciador de resultados $-\mathrm{y}$ de oportunidades para aprender- era el tipo de colegio según el NSE mayoritario de su alumnado, lo cual confirma la asociación entre segregación escolar y logros del aprendizaje.

Existe creciente información y discusión sobre la relación de la segregación escolar socioeconómica con las condiciones de educabilidad en las escuelas y los resultados del aprendizaje en algunas áreas curriculares. Sin embargo, se ha investigado menos acerca de la relación de la segregación escolar con las percepciones de la sociedad y de las familias sobre la segmentación de la oferta educativa. Tampoco, se ha estudiado mucho la relación de la segregación con el desarrollo de características individuales e interpersonales; las actitudes sociales de los estudiantes; y las dinámicas de la sociedad, tales como la cohesión social, la inclusión, la convivencia democrática y el conflicto.

En este artículo, se analiza y se discute evidencia disponible acerca de cómo la segregación socioeconómica de las escuelas podría estar operando como un mensaje tanto para los estudiantes, influyendo en sus percepciones, actitudes y conductas sociales, como para las familias y para la sociedad. El mensaje de la segregación no solo estaría implícito en la composición socioeconómica homogénea del alumnado en cada escuela, sino que se expresaría de manera concreta, objetiva y hasta brutal, a través de las desigualdades en la calidad de los procesos, las oportunidades y los resultados de la educación. Es decir, la calidad de la infraestructura escolar, los recursos y equipos, las prácticas de gestión y pedagógicas, las expectativas y el compromiso profesional de los maestros, el apoyo de los padres, la organización y el clima de la escuela son factores que caracterizan y diferencian a las escuelas dependiendo de su ubicación en la estructura social (Murillo y Carrillo, 2021; Balarin y Escudero, 2019).

El contexto cultural y político de la sociedad, que hace posible una estructura escolar segregada, le otorga a esta un significado que se transmite a todos los integrantes de cada comunidad escolar: directivos, docentes, padres de familia y estudiantes (Murillo y Carrillo, 2021). El propósito de este artículo es reflexionar sobre el sentido de ese significado transmitido, y su posible influencia sobre percepciones, actitudes y comportamientos, individuales y sociales. 
En esa línea, se revisa bibliografía para analizar y discutir si la segregación es el mensaje y si el contenido específico del mensaje depende de la ubicación de cada escuela en la pirámide estratificada del sistema escolar segregado. En la vida cotidiana, la sociedad distingue las escuelas para las personas más pobres de otras que acogen a los vulnerables; las que agrupan a los emergentes; las que reciben a las clases medias; $y$, finalmente, aquellas a las que solo tienen acceso los hijos de los ricos. Sin embargo, esta estructura de la oferta educativa segmentada por NSE es percibida como una característica "normal" de la realidad educativa nacional, y aceptada con indiferencia o con resignación por la mayor parte de la población.

\section{La segregación escolar como mensaje para las familias}

En el marco propuesto en este artículo, la ubicación de las escuelas en la estructura estratificada en función del NSE de sus alumnos sería un mensaje para todas las familias, tanto para aquellas que buscan en la educación la vía para reproducir sus privilegios y mantener su estatus social en la siguiente generación, como para quienes aspiran a ofrecer a sus hijos oportunidades de ascenso social eligiendo la escuela adecuada.

En su estudio sobre el tránsito de la escuela pública a la privada en el sector social emergente de Lima Metropolitana, Sanz (2015) encontró que las familias toman la decisión de migrar, porque perciben que la educación pública es de baja calidad y que la privada es mejor. La autora señala que esta percepción es parte de un sentido común instalado entre las familias, que ven en la escuela pública un servicio deficiente y, en consecuencia, el riesgo de fracasar en la vida adulta. La condición de privada conlleva para ellas el significado de mejor, a lo que se suma con frecuencia la idea de que el precio es señal de calidad. El tipo de gestión (estatal o privada) y el precio a pagar serían elementos del mensaje implícito de las escuelas a las familias.

La autora señala que la escuela es vista como un bien de consumo y que muchas familias desean pertenecer al sector que puede comprar educación privada para sus hijos:

el estatus y la exclusividad pueden distinguirse a lo largo del continuum entre las escuelas estatales y las escuelas privadas más exclusivas, pasando por los diferentes circuitos educativos que existen en el sistema y que impiden el contacto de los diferentes sectores sociales. (Sanz, 2015, p. 120)

Los mensajes para las familias de las distintas opciones de escuelas privadas incluirían - además de los precios- las condiciones de infraestructura, su localización y cercanía, la seguridad para los hijos y la ratio de alumnos por profesor, entre otros. Entre ellos, una señal muy importante sería la composición social de la escuela, que parece influir más que otros factores en la elección 
escolar de familias que buscan identificarse con otras iguales en estatus y diferenciarse de familias con menos recursos.

Sanz (2015) plantea que los criterios de elección de escuela de los dos grupos de familias emergentes observadas en su estudio ("precarios" y "consolidados") tienen coincidencias y diferencias. Los del sector más consolidado eligen escuelas privadas de mayor costo, no necesariamente cercanas a su domicilio; con infraestructura, equipamiento y servicios ad hoc para funcionar como colegio; ambiente cálido y personalizado; con 25 a 30 alumnos por salón; con métodos activos y centrados en los niños; que eviten el maltrato infantil; que consideren el desarrollo de las habilidades blandas requeridas para el éxito en la universidad y en el trabajo; y que traten a los padres como clientes con derecho a reclamar, y exigir cambios y mejoras para recibir calidad. En estas escuelas, ellos esperan encontrarse con familias similares en estatus o aspiraciones, con padres emprendedores y comprometidos con la educación de sus hijos.

El párrafo anterior describe el mensaje que los padres de familia emergentes consolidados esperarían encontrar en una escuela para elegirla. Con ese propósito, piden referencias y recomendaciones de amigos y familiares, visitan diferentes centros educativos, asisten a charlas informativas, realizan búsquedas en internet, se entrevistan con promotores y directores de las instituciones educativas. Es decir, leen en la realidad los mensajes de la oferta educativa accesible para elegir la escuela que mejor responda a sus expectativas y aspiraciones. Las escuelas más competitivas, por su parte, procuran incluir esos contenidos como parte de su realidad o de su imagen institucional. La homogeneidad social sería un elemento del mensaje para los padres que prefieren que sus hijos no se mezclen con niños diferentes a ellos en cuanto a procedencia social o cultural y experiencias de crianza.

En otro estudio sobre servicios educativos privados en el Perú (Balarin et al., 2018), se identificó que las escuelas urbanas de bajo costo suelen ser emprendimientos locales de promotores y directores que prometen una educación mejor a la de las escuelas públicas, a precios accesibles para familias que viven en condiciones de pobreza. Su mensaje implícito parecería enfatizar el valor de lo pequeño, lo cercano y lo personalizado; el trato directo y asertivo con cada familia; así como la ausencia de huelgas y de intermediaciones de asociaciones de padres de familia o de la burocracia estatal. Todo ello justificaría —en el imaginario de los padres de familia - un pago, a pesar de la precariedad de sus instalaciones, y las limitaciones profesionales y laborales de su personal. Quedan por investigar, no obstante, los posibles efectos de este mensaje a las familias sobre su valoración de la escuela y de la educación básica, sobre su voluntad para apoyar el proceso escolar, y sobre sus expectativas con respecto al potencial y el futuro de sus hijos, entre otros factores que se asocian con los logros del aprendizaje y el rendimiento en evaluaciones.

En los colegios conocidos como "preuniversitarios" y otros dirigidos a la clase media emergente o consolidada, el mensaje abierto o subliminal sería el siguiente: "educamos para el acceso a la universidad". La propuesta pedagógica 
y la gestión de esos colegios responden a ese objetivo y, también, serían parte del mensaje. Ofrecen una educación personalizada e integral, y tutoría que las familias de dichos sectores valoran. Aunque la denominación de "preuniversitarios" está prohibida, ese rasgo está implícito en el sentido común asociado a la elección de este tipo de colegios (Guzmán, 2016). Al prevalecer en la educación peruana las dinámicas de mercado, cada escuela privada procura alinearse con las expectativas de su correspondiente nicho de negocio, y también incluye la diferenciación social -que es inherente a la segregación-como parte de su imagen o mensaje. Como señala Miranda (2021), las familias de mayores ingresos optan por los colegios más caros no solamente para asegurar el acceso de sus hijos a una mejor educación, sino como estrategia de diferenciación social.

En general, la ubicación de cada institución educativa en la estructura piramidal estratificada y segregada que caracteriza al sistema escolar peruano podría ser reconocida como un mensaje para las familias. Esta incluiría información acerca de las condiciones y la calidad del servicio educativo, la composición social de sus estudiantes, y su posible eficacia como medio de ascenso social y de acceso a oportunidades para el futuro desarrollo personal y educativo de sus hijos, entre otros aspectos. La relación con un determinado tipo de escuela, cuando la imagen previa se confirma con los mensajes que provienen de la experiencia educativa cotidiana, probablemente retroalimenta las creencias sobre el sentido de la educación, y refuerza las expectativas de los padres con respecto al potencial de aprendizaje y de realización personal de sus hijos. Aún se requiere, sin embargo, de más investigación para confirmar la validez, y explorar la posible utilidad conceptual y política de esta interpretación.

\section{La segregación escolar como mensaje para los estudiantes}

Los estudiantes, por su parte, reciben el mensaje discriminador de la segregación escolar por NSE como experiencia vivencial cotidiana, como parte del currículo oculto, al margen de las intenciones del currículo prescrito y de los propósitos explícitos de los docentes. Así como las metodologías tradicionales de enseñanza son parte de un currículo oculto que legitima el autoritarismo, las prácticas antidemocráticas y ciertas representaciones sociales (Ames, 1989, y Eguren y De Belaunde, 2012, citados en Minedu, 2018a), la ubicación de la escuela en la estructura segregada y piramidal del sistema escolar podría conllevar implícito un mensaje que promueve determinadas representaciones sociales, actitudes y valores, que se desarrollarían como un sentido común. Dicho sentido común fungiría como orientador de la acción y de las interacciones de los individuos en la vida cotidiana en la sociedad adulta.

A continuación, se revisan algunas referencias de la bibliografía internacional sobre los efectos subjetivos de la segregación escolar, que han sido investigados más ampliamente en otros países. Así, por ejemplo, el 44 I Comisionado para los Derechos Humanos del Consejo de Europa (2017) señaló 
en su informe que el aislamiento de estudiantes gitanos nómades y migrantes debilita la cohesión social, reduce la tolerancia hacia la diversidad y el respeto a diferencias culturales y religiosas, y aumenta el racismo y la discriminación entre los jóvenes. ¿Cómo explicar estos efectos de la segregación escolar?

En Estados Unidos de América (EE.UU.), Mijs y Roe (2021) encontraron que la segregación socioeconómica perjudica la solidaridad, debilita la confianza y polariza la política, además de que erosiona las oportunidades para la movilidad social. Según los autores, la segregación esconde o normaliza las desigualdades, tanto para los alumnos ricos como para los pobres, puesto que no las experimentan en el día a día de la escuela. A partir de ello, también se reduce la preocupación de los políticos por las inequidades. Según estos autores, los jóvenes privilegiados crecen con una comprensión muy ingenua de la meritocracia americana.

Los mismos autores destacan que la segregación no solo limita el contacto de los estudiantes con aquellos de otros NSE, sino que también reduce su relación con adultos distintos a sus propios padres. Los estudiantes ricos casi nunca conocen un adulto padre de familia desempleado o vulnerable, y, en cambio, su contacto con adultos con alto nivel educativo es mucho mayor que el de los estudiantes pobres; estos últimos viven la experiencia contraria. Todos estos elementos - que forman parte del currículo oculto o implícito-, al parecer, inciden en la adopción de modelos y en la definición de expectativas, en especial durante la adolescencia.

De un modo similar, tras un año de observación e interacción con los estudiantes de un colegio segregado de élite en EE.UU., Khan (2011) concluyó que los estudiantes privilegiados creen que sus ventajas son producto de su esfuerzo y sus méritos, y que no son heredadas. Este sector piensa que, en la llamada sociedad abierta, hay ganadores y perdedores, y que eso depende de cada uno. El autor señala que, en realidad, los logros de estos estudiantes son producto de las condiciones de crianza, y que "donde la élite alega mérito, hay privilegios". La educación segregada, explica el autor, los lleva a desconocer o negar la existencia creciente de la desigualdad social y los privilegios.

La evidencia muestra que las desigualdades inherentes a un sistema escolar segregado por NSE tienen su origen en condición económica o educativa de las familias. Sin embargo, a menudo, las élites las atribuyen a una mayor capacidad o mérito académico de aquellos que son privilegiados. Sandel (2020) sostiene que la ética meritocrática fomenta actitudes negativas desde la perspectiva moral: entre los "ganadores", promueve la soberbia por supuestos logros personales, y, entre los "perdedores", propicia la humillación y el resentimiento por el fracaso, que atribuyen a sus propias debilidades individuales. Para este autor, estos sentimientos morales derivados de la segregación educativa constituyen el trasfondo de la actual polarización social y de la revuelta populista contra las élites en Estados Unidos de América.

Sandel agrega que a los ganadores el éxito "se les sube a la cabeza" y olvidan el carácter azaroso - o arbitrario — de la posición que ocupan; creen que se 
merecen el destino que les ha tocado y que aquellos menos privilegiados también se merecen el suyo. La idea de meritocracia - dice el autor-disminuye nuestra capacidad para concebirnos como seres que compartimos un destino común, y deja escaso margen a la solidaridad que puede surgir cuando reconocemos el carácter azaroso de nuestras aptitudes y fortunas. La segregación escolar por NSE sería un factor determinante de las diferencias en las condiciones y trayectorias educativas de "ganadores" y "perdedores". Sin embargo, cada vez más, estos últimos descubren que sus dificultades educativas y económicas no se deben a menores capacidades o esfuerzos, como pretende el discurso del mérito, sino a las desigualdades sociales que determinan desventajas desde el nacimiento y se reproducen en las escuelas segregadas. Sería conveniente investigar si ocurre una dinámica similar en el Perú, y cuáles serían sus efectos en el desarrollo personal y social.

En Chile, donde el sistema escolar también es muy segregado por niveles socioeconómicos (Carrasco et al., 2014), se ha identificado que las escuelas más inclusivas y menos segregadas benefician tanto a los estudiantes de mayor NSE como a los de bajos recursos, y no solo en su rendimiento académico. Estos últimos mejoran en su motivación, tienen expectativas más altas, amplían su experiencia de mundo y mejoran la autorregulación de su conducta. Por su parte, los estudiantes con mayores ventajas económicas consolidan sus aprendizajes, desarrollan actitudes colaborativas y enriquecen su discernimiento moral: llegan a la vida laboral y democrática más tolerantes y abiertos.

En un estudio realizado en el Perú, Carrillo (2021) también ha resaltado las ventajas de la diversidad de los grupos de aula y de escuela, tanto en función del rendimiento académico como de las emociones y afectos, experiencias, ritmos y estilos de aprendizaje, la convivencia y el manejo de conflictos. Varias de esas ventajas desaparecen cuando las escuelas son segregadas y socialmente homogéneas, lo cual sugiere la posible existencia de un mensaje o contenido oculto según el tipo - segregado o no- de las escuelas.

Una investigación de Cueto et al. (2016), a partir de datos del estudio longitudinal del Proyecto Niños del Milenio, mostró que la composición social segregada de las escuelas reduce el sentido de pertenencia de los estudiantes a sus respectivas escuelas. Este también podría ser un efecto del mensaje o el currículo oculto que está implícito en el carácter segregado de cada escuela. El sentido de pertenencia, según Tacca et al. (2020), está asociado al autoconcepto. Este, a su vez, se relaciona con la autoestima, la inteligencia emocional, el rendimiento académico, la violencia escolar y el consumo de sustancias psicoactivas, la relación con los pares y el comportamiento social de los adolescentes, los que también podrían estar asociados con la segregación. La socialización en escuelas segregadas también limita el desarrollo de la empatía y la valoración de los otros diferentes (Miranda, 2021).

Una evaluación sobre Cívica y Ciudadanía realizada por la Unidad de Medición de la Calidad (Minedu, 2016) reveló los siguientes resultados: en general, solo $34,8 \%$ de los estudiantes de segundo de secundaria reconocieron 
la democracia como sistema político y apenas $8 \%$ de alumnos consiguieron evaluar posiciones políticas o leyes en función de principios democráticos y de la búsqueda del bien común. En el informe, destaca que el desempeño en la prueba mejora a medida que se eleva el nivel socioeconómico de las escuelas. Cabe preguntar si la identificación con la democracia, la percepción de inclusión social, y la confianza en el Estado y en el sistema político vigente están condicionados en alguna medida por los mensajes de desigualdad y discriminación asociados con el carácter segregado de las escuelas.

Otro estudio de la Oficina de Medición de la Calidad de Aprendizajes (Minedu, 2018b) utilizó dilemas — situaciones hipotéticas de la vida ciudadana- para estudiar la elección de valores ciudadanos (solidaridad, respeto a la ley, defensa del estado de derecho y de la libertad individual, entre otros) en una muestra representativa nacional de estudiantes de sexto grado de primaria. En este caso, también, se evidenció que los estudiantes de nivel socioeconómico menor tienden a optar por valores asociados a beneficios concretos, y a las necesidades económicas inmediatas de personas y comunidades. En cambio, las respuestas de aquellos de nivel socioeconómico mayor tienden a optar por valores asociados al respeto a las leyes y los principios de una sociedad democrática, como la defensa de la libertad individual. Cabe preguntar si la relación entre NSE y los valores está mediada por el agrupamiento de poblaciones socialmente homogéneas en colegios segregados.

En algunas investigaciones, se ha encontrado que la disposición de los jóvenes hacia la democracia no parece estar asociada con el número de años de escolaridad ni con la calidad de la educación recibida, y tampoco con la introducción de contenidos curriculares explícitos sobre democracia, o la existencia formal de instancias de participación estudiantil, salvo cuando estas permiten actuar con autonomía y de manera auténtica en asuntos relevantes de la vida escolar cotidiana, según refiere Stojnic (2020). Este autor recomienda reflexionar sobre la escuela como institución social, sus formas de organización y funcionamiento, y la cultura pública que reproduce desde la experiencia porque, al parecer, el desarrollo de la cultura democrática se relaciona más con la composición social heterogénea de la escuela y la existencia de experiencias auténticas de participación en las decisiones escolares que con la enseñanza de conceptos acerca de la democracia. De ser así, el carácter segregado de las escuelas sería una barrera para el desarrollo de valores y actitudes democráticos, en la medida que estaría provocando vivencias cotidianas de exclusión, discriminación y desigualdad, que son contrarias a la democracia.

Es necesario ampliar y profundizar mucho más el estudio de la relación entre la segregación escolar por NSE y el desarrollo de actitudes sociales y democráticas de los estudiantes. Como señalan Pease et al. (s.f.) en un informe del proyecto "Ser Adolescente en el Perú", aún es poco lo que se sabe sobre los adolescentes peruanos, cómo son y cómo aprenden, cómo son sus vivencias, cómo representan la realidad peruana y su propia relación con ella, y otros aspectos de su subjetividad. Los autores añaden la importancia de investigar 
las relaciones particulares que las y los adolescentes de diversas realidades construyen con la escuela secundaria, reconocer a partir de sus voces qué elementos de la cultura escolar afectan de manera negativa sus aprendizajes y en qué medida sus expectativas de escolaridad se encuentran cubiertas... (s.f., p. 4).

La realidad de la segregación en las escuelas a las que asisten, y su relación con el desarrollo personal y social de los adolescentes también deberían ser objeto de futuras investigaciones. Como plantean Pease et al. (s.f.), es más importante la forma como las personas perciben su contexto que la manera en que son observadas objetivamente en la realidad. Por ello, se debería estudiar la percepción de los adolescentes sobre la calidad de la educación que reciben en contraste con la que reciben los adolescentes de otras clases sociales, así como la valoración que tienen de su propia institución educativa en comparación con otras ubicadas en otros niveles del sistema educativo estratificado por NSE. En ese marco, hace falta indagar qué significado tiene la segregación escolar socioeconómica para los y las adolescentes matriculados en instituciones educativas ubicadas en los distintos niveles del sistema escolar segregado y piramidal. Al respecto, Acosta señala (2017):

Toda oferta que diferencia grupos (por escuela o dentro de la escuela) también produce estigmas y las escuelas secundarias, en particular, tienen una larga historia en este tipo de dinámica...; ese sería el efecto de las llamadas "escuelas para pobres" (p. 217).

En este mismo sentido, Carrillo (2021) advierte que la experiencia escolar segregada puede asociarse a la frustración juvenil, y a la formación de grupos que reproducen la desigualdad y la pobreza familiar a través de comportamientos de imitación negativos.

En síntesis, se propone como hipótesis que la ubicación de cada escuela en la estructura piramidal segregada y desigual del sistema escolar sea considerada como un mensaje o contenido curricular oculto, que se asocia con el desarrollo de valores y actitudes opuestos a una convivencia democrática y una ciudadanía respetuosa de la diversidad. En contraste con los propósitos inclusivos e integradores de las normas y el currículo oficial, la experiencia de segregación parece fomentar la intolerancia, la discriminación, prejuicios sobre capacidades y méritos, la soberbia de unos y el resentimiento de otros, poco desarrollo del sentido de pertenencia, la autoestima o la empatía, diferentes grados de identificación con la democracia y el estado de derecho, la estigmatización de la pobreza, y desigualdades en las expectativas personales de los estudiantes. Las investigaciones futuras podrían confirmar la existencia y la importancia de estas relaciones como insumos para los diagnósticos y las propuestas de transformación de la educación peruana. 


\section{La segregación escolar y la convivencia social democrática}

Por último, otro campo en que es necesario profundizar en el Perú corresponde a la relación entre la segregación escolar por NSE, y el desarrollo de la cohesión social y la democracia. Balarin y Escudero (2019) revisaron algunos estudios nacionales e internacionales, y concluyeron que esta situación de la educación repercute negativamente en la sociedad, pues impide que todos los estudiantes desarrollen valores ciudadanos compartidos, y se reconozcan con los mismos derechos y deberes. Siguiendo a Nussbaum (2003), y Wilkinson y Pickett (2009), las autoras indican que "al limitar la igualdad de oportunidades, la segregación educativa puede también tener una influencia negativa en el bienestar de la sociedad" (Balarin y Escudero, 2019, p. 11). Asimismo, señalan que la segregación limita la capacidad de las escuelas para actuar como espacios sociales integradores, y desempeñar un rol en la generación de cohesión social y en la promoción de una ciudadanía democrática.

Murillo y Carrillo (2021) también recuperaron conclusiones de estudios que han mostrado que la segregación escolar no solo afecta la posibilidad de asegurar a los estudiantes la igualdad de oportunidades en el logro de objetivos académicos, sino que, además, impide garantizar la calidad y la riqueza de la experiencia formativa para el logro de la cohesión social. En una línea similar, Cueto (2021) plantea que la alta segregación por NSE que caracteriza a la educación peruana puede tener implicancias para la integración de grupos sociales que no tienen oportunidades de encontrarse e interactuar en las escuelas.

Miranda (2021) afirma que el alto grado de la segregación escolar socioeconómica en el Perú afecta negativamente la formación de la ciudadanía, e impide la consolidación de una cultura democrática e inclusiva, que valore la diversidad y fomente la participación activa y responsable, en condiciones de igualdad y de colaboración entre grupos diferentes. La segregación escolar distorsiona el proceso de los estudiantes de acopio del capital social vinculante y del capital social puente. Según la autora, estos refieren a las relaciones dentro de una comunidad de iguales - en el primer caso- y al establecimiento de vínculos entre grupos de diferentes comunidades - en el segundo caso-. Las escuelas homogéneas refuerzan la relación entre próximos y reafirman identidades locales, pero no facilitan el tránsito a la integración en una mirada plural, que caracterizaría a una identidad nacional. Por el contrario, este tipo de instituciones aumenta la desconfianza entre comunidades separadas y diferentes, lo cual agrava los conflictos sociales.

En nuestro país, el Proyecto Educativo Nacional (PEN) al 2036 —elaborado por el Consejo Nacional de Educación (2020) y aprobado como política de Estado- también hace eco de la evidencia disponible al señalar que la profunda segregación social escolar existente niega la posibilidad de que el sistema educativo peruano cumpla con generar cohesión social en un entorno de respeto y valoración mutuos. Además, ello afecta negativamente los aprendizajes de los estudiantes, especialmente, de aquellos en desventaja. En las propuestas 
del PEN al 2036, se destaca la ciudadanía plena, igualitaria e inclusiva como finalidad de la educación nacional, así como se manifiesta que el sistema educativo debe promover "el encuentro entre las personas en toda la riqueza de su diversidad" (p. 74). Al ser una barrera que impide el logro de los propósitos democráticos del sistema educativo y del país, la eliminación de la segregación escolar socioeconómica debería ser reconocida como uno de los principales desafíos, y una de las tareas prioritarias a ser emprendidas desde las políticas y desde la gestión de la educación en el Perú.

\section{Comentarios finales}

La revisión de la relevancia de la segregación escolar sistémica y de los mensajes que estaría entregando el carácter segregado de las escuelas a las familias y a los estudiantes, así como de sus posibles efectos en la configuración de la convivencia social en democracia, muestra algunos indicios y datos significativos. No obstante, también abre un conjunto de nuevas preguntas. El desarrollo de la investigación para responder a esas y otras interrogantes sobre el tema podría enriquecer el conocimiento sobre este importante aspecto de la realidad educativa, y contribuir a la formulación y ejecución de políticas públicas efectivas para la transformación democrática de la educación.

$\mathrm{Si}$, como parece, la selección y la segregación de los estudiantes en escuelas separadas, homogéneas en su composición social, y desiguales entre unas y otras - en función del nivel socioeconómico de sus familias- conlleva mensajes implícitos que afectan a las comunidades educativas de todos los niveles socioeconómicos, también se podría considerar a estos mensajes como contenidos relevantes del currículo oculto vivencial que cada institución educativa, y el sistema escolar en su conjunto, estarían aplicando a los estudiantes de manera no intencionada. De este modo, se podría estar afectando negativamente el desarrollo de la identidad personal y social, el sentido de pertenencia a una comunidad diversa y compleja, la valoración de la diversidad social y cultural, así como la conformación de una ciudadanía democrática y la cohesión social.

Siguiendo a Giroux (1997), se puede asumir que el contenido del currículo oculto -el carácter segregado y desigual de la escuela y su ubicación en la estructura piramidal del sistema escolar- probablemente contribuye a la perpetuación de las desigualdades económicas, de las brechas y de la desintegración social, así como a la concentración del poder político. Todo ello, de ser el caso, se impondría y prevalecería sobre las buenas intenciones democratizadoras declaradas por el sistema educativo. Así, neutralizaría la eficacia de los contenidos discursivos sobre igualdad, equidad, ciudadanía, e inclusión previstos en los lineamientos del currículo escolar oficial y en los documentos de política educativa.

Finalmente, el reconocimiento del impacto negativo de la segregación escolar socioeconómica - tanto en términos objetivos como subjetivos, indi-

viduales como sociales - y de la urgencia de revertir su creciente predominio 
como característica del sistema educativo peruano exige el diseño y el desarrollo de políticas que promuevan la multiplicación de escuelas públicas integradoras y heterogéneas en su composición social y cultural, gratuitas, no selectivas y atractivas por su buena calidad (Bello, 2019). Si bien son necesarias las estrategias focalizadas en la mejora de la atención educativa diferenciada y específica para poblaciones discriminadas y vulnerables, estas no son suficientes para lograr un sistema educativo socialmente inclusivo; con mejores resultados del aprendizaje; con equidad, cohesión social y valoración de la diversidad; que promueva y desarrolle una convivencia ciudadana democrática. Para lograr este propósito, hacen falta escuelas en las que todas las clases sociales se encuentren en la experiencia educativa cotidiana y aprendan a vivir juntas. 


\section{Referencias bibliográficas}

Acosta, F. (2017). Segmentación y sistemas educativos: un análisis a través de la comparación de los cambios para la escuela secundaria en Europa y América Latina. Revista Española de Educación Comparada, 29 (enerojunio), 202-219.

Balarin, M. y Escudero, A. (2019). El desgobierno del mercado educativo y la intensificación de la segregación escolar socioeconómica en el Perú. Documentos de Investigación № 101. Grade.

Balarin, M., Kitmang, J., Ñopo, H. y Rodríguez, M. F. (2018). Mercado privado, consecuencias públicas: los servicios educativos de provisión privada en el Perú. Documentos de Investigación. Grade.

Bello, M. (2019). Ciudadanía y desigualdad familiar, escolar y urbana en Lima Metropolitana. La Mula Blogs. https://manuelbello.lamula. pe/2019/11/21 ciudadanía-y-desigualdad-familiar-escolar-y-urbana-enlima-metropolitana/mbellod/

Bello, M. y Villarán, V. (2004). Educación, reformas y equidad en los países de los Andes y Cono Sur: dos escenarios en el Perú. IIPE-Unesco.

Benavides, M., León, J. y Etesse, M. (2014). Desigualdades educativas y segregación en el sistema educativo peruano. Una mirada comparativa de las pruebas PISA 2000 y 2009. Avances de Investigación 15. Grade.

Carrasco, A., Contreras, D., Elacqua, G., Flores, C., Mizala, A., Santos, H., Torche, F. y Valenzuela, J. P. (13 de mayo de 2014). Hacia un sistema escolar más inclusivo. El Mercurio Blogs.

Carrillo, S. (2021). Ni juntos ni revueltos. La segregación escolar como uno de los grandes desafíos del Bicentenario. En C. Guadalupe (Ed.), La educación peruana más allá del bicentenario: nuevos rumbos. Universidad del Pacífico.

Comisionado para los Derechos Humanos del Consejo de Europa (2017). Fighting School Segregation in Europe Through Inclusive Education: A Position Paper. Comisionado para los Derechos Humanos, Consejo de Europa.

Consejo Nacional de Educación [CNE] (2020). Proyecto Educativo Nacional al 2036, el reto de la ciudadanía plena. Ministerio de Educación.

Cueto, S. (2021). Las políticas y programas que no se diseñan explícitamente para promover equidad aumentan la inequidad. Una hipótesis para el Perú. En C. Guadalupe (Ed.), La educación peruana más allá del bicentenario: nuevos rumbos. Universidad del Pacífico.

Cueto, S., Miranda, A. y Vásquez, M. C. (2016). Inequidades en la educación. Investigación para el desarrollo en el Perú: once balances (pp. 55-108). Grade. 
Giroux, H. (1997). Los profesores como intelectuales. Paidós.

Guzmán, M. F. (2016). En verdad es bien matado: el papel de los profesores y tutores en la formación de estudiantes en un colegio preuniversitario en Villa El Salvador [Tesis para optar el título de Licenciada en Antropología]. Pontificia Universidad Católica del Perú.

Khan, S. (2011). Privilegios, la construcción de un adolescente de élite. Princeton University Press.

Machado, A. L. (2004). Presentación. En M. Benavides, A. Maz, E. Castro y R. Blanco (Eds.). La educación de niños con talento en Iberoamérica (pp. 9-13). Orealc / Unesco.

McLuhan, M. (1996). Comprender los medios de comunicación. Las extensiones del ser humano. Paidós.

Mijs, J. J. B. y Roe, E. L. (2021). Is America Coming Apart? Socioeconomic segregation in neighborhoods, schools, workplaces, and social networks, 1970-2020. WILEY.

Ministerio de Educación (2016). Estudio Internacional de Cívica y CiudadaníaICCS 2016: Resultados del Perú. Unidad de Medición de la Calidad. http:// umc.minedu.gob.pe/wp-content/uploads/2017/11/Presentaci\%C3\%B3nde-resultados-ICCS-2016.pdf

Ministerio de Educación (2018a). Currículo Nacional de Educación Básica. Ministerio de Educación. http://www.minedu.gob.pe/curriculo/pdf/ curriculo-nacional-2016-2.pdf

Ministerio de Educación (2018b). Dilemas, valores y ciudadanía. Una aproximación a la elección de valores ciudadanos en estudiantes de sexto grado de primaria. Oficina de Medición de la Calidad de los Aprendizajes.

Ministerio de Educación (2019). Equidad y oportunidades de aprendizaje en 2 do grado de secundaria. Evidencias de la ECE 2016. Oficina de Medición de la Calidad de los Aprendizajes. http://umc.minedu.gob.pe/equidad-yoportunidades-de-aprendizaje-en-2-grado-de-secundaria-evidencias-dela-ece-2016/

Miranda, L. (2021). Tensiones entre segregación escolar y desarrollo ciudadano. En C. Guadalupe (Ed.), La educación peruana más allá del bicentenario: nuevos rumbos. Universidad del Pacífico.

Murillo, F. J. y Carrillo, S. (2020). Segregación escolar por nivel socioeconómico en educación secundaria en Perú y sus regiones. Revista Peruana de Investigación Educativa, 12, 7-32.

Murillo, F. J., y Carrillo, S. (2021). Incidencia de la segregación escolar por nivel socioeconómico en el rendimiento académico. Un estudio desde Perú. Archivos Analíticos de Políticas Educativas, 29(49). https://doi. org/10.14507/epaa.29.5129 
Murillo, F. J., Duk, C. y Martínez Garrido, C. (2018). Evolución de la segregación socioeconómica de las escuelas de América Latina. Estudios Pedagógicos 44(1), 157-179.

Pease, M. A., Guillén, H., De La Torre-Bueno, S., Urbano, E., Araníbar, C. y Rengifo, F. (s.f.). Nuestra deuda con la adolescencia. Proyecto "Ser adolescente en el Perú". Convenio Unicef-Pucp.

Sandel, M. J. (2020) La tiranía del mérito. ¿Qué ha sido del bien común? (Spanish Edition). Penguin Random House Grupo Editorial. https://a.co/5XPHaRj

Sanz, P. (2015). El tránsito de la escuela pública a la privada en el sector emergente de Lima Metropolitana: ¿Buscando mejor calidad? Revista Peruana de Investigación Educativa 7, 95-125.

Stojnic, L. (2020). Participación estudiantil, institucionalidad escolar y ciudadanía democrática: desafíos pendientes desde la experiencia peruana. Revista Internacional de Educación para la Justicia Social, 2020, 9(2), 49-70. https://revistas.uam.es/riejs/article/view/riejs2020_9_2_003

Tacca Huamán, D. R., Cuarez Cordero, R. y Quispe Huaycho, R. (2020). Habilidades Sociales, Autoconcepto y Autoestima en Adolescentes Peruanos de Educación Secundaria. International Journal of Sociology of Education, 9(3) octubre, 293-324. http://doi.org/10.17583/rise.2020.5186 